\title{
On the Azimuthal Mode Propagation in AXISYMMETRIC DUCT FLOWS
}

\author{
X. D. Li* C. Schemel ${ }^{\dagger}, \quad$ U. Michel ${ }^{\ddagger}, \quad$ F. Thiele ${ }^{\S}$ \\ ${ }^{*}$ Beijing University of Aeronautics \& Astronautics, Xueyuan Street 37, 100083, Beijing, PRC \\ ${ }^{\ddagger}$ DLR Institute of Propulsion Technology, Mueller-Breslau-Strasse 8, D-10623, Berlin, Germany \\ ${ }^{\dagger}$ Technical University of Berlin, Strasse des 17. Juni 135, D-10623, Berlin, Germany
}

\begin{abstract}
Starting from the 3D linearized Euler equations and decomposing the 3D perturbation quantities into a Fourier series in the azimuthal direction, a set of 2.5D linearized disturbance equations is derived which are valid for each azimuthal mode of fluctuation. The derivation is based on an axisymmetric mean flow and axisymmetric acoustic boundary conditions. A Computational Aeroacoustics (CAA) approach is applied to solve the 2.5D equation system. A fourth-order Dispersion-Relation-Preserving finite difference scheme is implemented for spatial discretization, whereas a $2 \mathrm{~N}$ storage form Low Dissipation and Low Dispersion Runge-Kutta scheme is applied for time integration. Appropriate boundary conditions are prescribed at different boundary regions. The numerical procedure is firstly validated by a straight circular pipe and a straight annular duct subjected on subsonic uniform mean flows. Numerical results show very good agreement with the analytical solutions. Further numerical example is presented for an axisymmetric duct inlet with an aero-engine like geometry including a spinner inside. The aeroacoustic computation is based on an inviscid mean flow calculated by a 2nd order CFD solver. The CAA solutions agree rather well with the finite element results of Eversman as well as the semi-analytical multiple-scales solutions of Rienstra. In particular, a cut-on cut-off transition case has been simulated. This reveals the feasibility of the proposed theory and solution procedure.
\end{abstract}

\section{$\underline{\text { INTRODUCTION }}$}

Fan noise is known as the dominant noise component of modern high bypass ratio aero-engines. To accurately evaluate the sound propagation in an aeroengine inlet and its far field radiation, the prediction methods are required to be capable of handling complex geometry, including the spinners cross section and accounting for basic mean flow effects. In general, fully three dimensional numerical methods are required for the description of the sound propagation in a duct. ${ }^{1234}$ The main disadvantage is that the 3D methods have high costs in both time consumption as well as the computational resources demand, especially for high frequency cases.

This paper concerns small amplitude sound propagation in axisymmetric duct flows. The three-dimensional linearized Euler equations in a cylindrical coordinate system $(x, r, \phi)$ can be chosen to describe this problem. However, under the assumption of an axisymmetric mean flow and axisymmetric acoustic boundary conditions, the three-

\footnotetext{
* Associate professor, Department of Jet Propulsion, Member AIAA.

${ }^{\dagger}$ Graduate student, Hermann-Föttinger-Institute for Fluid Mechanics.

$¥$ Senior scientist, Section Turbulence Research Berlin.

$\S$ Professor, Hermann-Foettinger-Institute for Fluid Mechanics.

Copyright (C) 2002 by X. D. Li, C. Schemel, U. Michel, F. Thiele. Published by the American Institute of Aeronautics and Astronautics, Inc. with permission.
}

dimensional fluctuating quantities can be decomposed into a Fourier series in the azimuthal direction. This yields a system of independent two-dimensional $(x, r)$ differential equations for each azimuthal mode $m$ of the fluctuations. Since tone noise in the inlet of aircraft engines is generally dominated by only a few components at the blade passing frequency and its harmonics, it is computationally much more efficient to treat these few two-dimensional Fourier components rather than to solve the full three-dimensional system. In fact, the Fourier decomposition technique has found various applications in axisymmetric duct acoustics. For example, it was employed in the Finite Element Method (FEM) of Eversman, etc. ${ }^{5}$ as well as the multiplescales (MS) method of Rienstra. ${ }^{6}$

In this paper, a 2.5D CAA approach is given, which is numerically based on our previous 2D CAA procedure. ${ }^{7}$ This procedure is firstly validated in comparison with the analytical solutions in a circular and an annular duct flow. Furthermore some numerical results are given for an aeroengine inlet duct geometry with flow and compared with the results of the FEM and MS methods. ${ }^{8}$ Finally a turning plane case is simulated and analyzed. 


\section{MATHEMATICAL MODEL}

Concerning small amplitude sound wave propagation in a cylindrical duct with subsonic inviscous mean flow, the starting point to derive the 2.5D nondimensionless governing equations are the linearized 3D Euler equations (LEE), as shown below.

$$
\begin{aligned}
& \frac{\partial \varrho^{\prime}}{\partial t}+\bar{\varrho} \frac{\partial u^{\prime}}{\partial x}+\bar{U} \frac{\partial \varrho^{\prime}}{\partial x}+\bar{\varrho} \frac{\partial v^{\prime}}{\partial r}+\bar{V} \frac{\partial \varrho^{\prime}}{\partial r} \\
& +\frac{1}{r} \bar{\varrho} \frac{\partial w^{\prime}}{\partial \phi}+\frac{1}{r} \bar{W} \frac{\partial \varrho^{\prime}}{\partial \phi}+u^{\prime} \frac{\partial \bar{\varrho}}{\partial x}+\varrho^{\prime} \frac{\partial \bar{U}}{\partial x} \\
& +v^{\prime} \frac{\partial \bar{\varrho}}{\partial r}+\varrho^{\prime} \frac{\partial \bar{V}}{\partial r}+\frac{1}{r} w^{\prime} \frac{\partial \bar{\varrho}}{\partial \phi}+\frac{1}{r} \varrho^{\prime} \frac{\partial \bar{W}}{\partial \phi} \\
& +\frac{1}{r}\left(\varrho v^{\prime}+\varrho^{\prime} \bar{V}\right)=0 \\
& \frac{\partial u^{\prime}}{\partial t}+\bar{U} \frac{\partial u^{\prime}}{\partial x}+\frac{1}{\bar{\varrho}} \frac{\partial p^{\prime}}{\partial x}+\bar{V} \frac{\partial u^{\prime}}{\partial r}+\frac{1}{r} \bar{W} \frac{\partial u^{\prime}}{\partial \phi} \\
& +\left(u^{\prime}+\bar{U} \frac{\varrho^{\prime}}{\bar{\varrho}}\right) \frac{\partial \bar{U}}{\partial x}+\left(v^{\prime}+\bar{V} \frac{\varrho^{\prime}}{\varrho}\right) \frac{\partial \bar{U}}{\partial r} \\
& +\frac{1}{r}\left(w^{\prime}+\bar{W} \frac{\varrho^{\prime}}{\bar{\varrho}}\right) \frac{\partial \bar{U}}{\partial \phi}=0 \\
& \frac{\partial v^{\prime}}{\partial t}+\bar{U} \frac{\partial v^{\prime}}{\partial x}+\bar{V} \frac{\partial v^{\prime}}{\partial r}+\frac{1}{\bar{\varrho}} \frac{\partial p^{\prime}}{\partial r}+\frac{1}{r} \bar{W} \frac{\partial v^{\prime}}{\partial \phi} \\
& +\left(u^{\prime}+\bar{U} \frac{\varrho^{\prime}}{\bar{\varrho}}\right) \frac{\partial \bar{V}}{\partial x}+\left(v^{\prime}+\bar{V} \frac{\varrho^{\prime}}{\varrho}\right) \frac{\partial \bar{V}}{\partial r} \\
& +\frac{1}{r}\left(w^{\prime}+\bar{W} \frac{\varrho^{\prime}}{\bar{\varrho}}\right) \frac{\partial \bar{V}}{\partial \phi} \\
& -\frac{1}{r}\left(2 \bar{W} w^{\prime}+\frac{\varrho^{\prime}}{\underline{\varrho}} \bar{W}^{2}\right)=0 \\
& \frac{\partial w^{\prime}}{\partial t}+\bar{U} \frac{\partial w^{\prime}}{\partial x}+\bar{V} \frac{\partial w^{\prime}}{\partial r}+\frac{1}{r}\left(\bar{W} \frac{\partial v^{\prime}}{\partial \phi}+\frac{1}{\bar{\varrho}} \frac{\partial p^{\prime}}{\partial \phi}\right) \\
& +\left(u^{\prime}+\bar{U} \frac{\varrho^{\prime}}{\bar{\varrho}}\right) \frac{\partial \bar{W}}{\partial x}+\left(v^{\prime}+\bar{V} \frac{\varrho^{\prime}}{\varrho}\right) \frac{\partial \bar{W}}{\partial r} \\
& +\frac{1}{r}\left(w^{\prime}+\bar{W} \frac{\varrho^{\prime}}{\bar{\varrho}}\right) \frac{\partial \bar{W}}{\partial \phi} \\
& +\frac{1}{r}\left(\bar{W} v^{\prime}+\bar{V} w^{\prime}+\frac{\varrho^{\prime}}{\varrho} \bar{\varrho} \bar{W}\right)=0
\end{aligned}
$$

The connection of pressure and density is based on the isentropic law:

$$
p^{\prime}=\gamma \frac{\bar{p}}{\bar{\varrho}} \varrho^{\prime}
$$

The local values of the mean flow field are marked by a bar and the sound field by a prime. The length scales are nondimensionalized by the typical radius of the duct, the velocity components by the far field speed of sound, the density by the far field density and the pressure by the product of squared far field speed of sound and density. The nondimensionalization is consolidated in the following table 1.

\section{Table 1 Nondimensionalization}

\begin{tabular}{ccc} 
definition & nondimensianal quantity & reference \\
\hline length & $x, r$ & $R$ \\
velocity & $u^{\prime}, v^{\prime}, w^{\prime}, \bar{U}, \bar{V}, \bar{W}$ & $a_{\infty}$ \\
time & $t$ & $\frac{R}{a_{\infty}}$ \\
density & $\varrho^{\prime}, \bar{\varrho}$ & $\varrho_{\infty}$ \\
pressure & $p^{\prime}, \bar{p}$ & $\varrho_{\infty} a_{r}^{2}$ \\
\hline
\end{tabular}

Considering an axisymmetric duct geometry with axisymmetric mean flow, the dependency of the sound field on the circumferential coordinate $\phi$ is described analytically, e.g. for the pressure:

$$
p^{\prime}(t, x, r, \phi)=p_{m}^{\prime}(t, x, r) e^{-i m \phi}
$$

Now a Fourier series decomposition of the differential equations for the sound field is employed in the azimuthal direction.

$$
\int_{0}^{2 \pi} \sum_{m=-\infty}^{\infty} \theta_{m}(x, r, t) e^{-i m \phi} e^{i k \phi} d \phi=0
$$

Wherein $\theta$ denotes the rewritten complex differential equation which is independent of $\phi$.

To take advantage of this approach, it is assumed that only a finite number of azimuthal modes are of interest for the problem, therefore the summation will not approach to infinity. In this case the relations of orthogonality for the products of the complex exponential function lead to a decoupled set of equations for each azimuthal mode. All these equations for all azimuthal modes together describe the complete 3D sound field, hence this formulation is called 2.5D. This means, that the azimuthal modes have to be computed one by one and then can be merged to a $3 \mathrm{D}$ sound field.

Based on the assumptions made above and additionally restricted to a mean flow without swirl $(\bar{W}=0)$, the $2.5 \mathrm{D}$ governing equations for a single mode can be derived and written as:

$$
\begin{array}{r}
\frac{\partial \varrho_{m}^{\prime}}{\partial t}+\bar{\varrho} \frac{\partial u_{m}^{\prime}}{\partial x}+\bar{U} \frac{\partial \varrho_{m}^{\prime}}{\partial x}+\bar{\varrho} \frac{\partial v_{m}^{\prime}}{\partial r}+\bar{V} \frac{\partial \varrho_{m}^{\prime}}{\partial r} \\
+u_{m}^{\prime} \frac{\partial \bar{\varrho}}{\partial x}+\varrho_{m}^{\prime} \frac{\partial \bar{U}}{\partial x}+v_{m}^{\prime} \frac{\partial \bar{\varrho}}{\partial r}+\varrho_{m}^{\prime} \frac{\partial \bar{V}}{\partial r} \\
+\frac{1}{r} \bar{\varrho}\left(v_{m}^{\prime}-i m w_{m}^{\prime}\right)+\frac{1}{r} \varrho_{m}^{\prime} \bar{V}=0
\end{array}
$$




$$
\begin{gathered}
\frac{\partial u_{m}^{\prime}}{\partial t}+\bar{U} \frac{\partial u_{m}^{\prime}}{\partial x}+\bar{V} \frac{\partial u_{m}^{\prime}}{\partial r}+\left(u_{m}^{\prime}+\frac{\varrho_{m}^{\prime}}{\bar{\varrho}} \bar{U}\right) \frac{\partial \bar{U}}{\partial x} \\
+\left(v_{m}^{\prime}+\frac{\varrho_{m}^{\prime}}{\bar{\varrho}} \bar{V}\right) \frac{\partial \bar{U}}{\partial r}+\frac{1}{\bar{\varrho}} \frac{\partial p_{m}^{\prime}}{\partial x}=0 \\
\frac{\partial v_{m}^{\prime}}{\partial t}+\bar{U} \frac{\partial v_{m}^{\prime}}{\partial x}+\bar{V} \frac{\partial v_{m}^{\prime}}{\partial r}+\left(u_{m}^{\prime}+\frac{\varrho_{m}^{\prime}}{\bar{\varrho}} \bar{U}\right) \frac{\partial \bar{V}}{\partial x} \\
+\left(v_{m}^{\prime}+\frac{\varrho_{m}^{\prime}}{\bar{\varrho}} \bar{V}\right) \frac{\partial \bar{V}}{\partial r}+\frac{1}{\bar{\varrho}} \frac{\partial p_{m}^{\prime}}{\partial r}=0 \\
\frac{\partial w_{m}^{\prime}}{\partial t}+\bar{U} \frac{\partial w_{m}^{\prime}}{\partial x}+\bar{V} \frac{\partial w_{m}^{\prime}}{\partial r}+\frac{1}{r} \bar{V} w_{m}^{\prime} \\
-\frac{1}{\bar{\varrho}} \frac{i m}{r} p_{m}^{\prime}=0
\end{gathered}
$$

The closing equation based on the isentropic law connects the complex pressure fluctuation to complex density fluctuation:

$$
p_{m}^{\prime}=\gamma \frac{\bar{p}}{\bar{\varrho}} \varrho_{m}^{\prime}
$$

In summary, the resulting method consists of the following three steps:

- Fourier decomposition of the 3D source into azimuthal modes

- CAA computation of the sound propagation for each mode

- reconstruction of the computed solutions to a 3D sound field with the assumed dependency of $m \phi$

\section{NUMERICAL ALGORITHM}

\section{DISCRETIZATION SCHEME}

A $2 \mathrm{D} \mathrm{CAA}$ code $^{7}$ is extended to solve the $2.5 \mathrm{D}$ equations, Eq. (8) Eq. (12). A Dispersion-Relation-Preserving (DRP) scheme ${ }^{9}$ is employed for space discretization. The solution is advanced in time by a Low-Dissipation and Low-Dispersion Runge-Kutta (LDDRK) scheme ${ }^{10}$ in the $2 \mathrm{~N}$ storage form. ${ }^{11}$ In order to obtain high quality numerical solutions selective artificial damping is adopted as a background damping to eliminate short wavelength spurious waves. ${ }^{12}$ This damping is also employed around special points, where the grid is stretched or the boundary condition is varying. This is especially crucial for the points around the spinners tip where the spinners inner hard wall meets the axis.

\section{BOUNDARY CONDITIONS}

Different boundary region and flow physics require appropriate boundary conditions (BCs), respectively. Detailed descriptions are introduced as below (see Fig. 1 and Fig. 4):

\section{Inflow Boundary}

In this paper, we focus on upstream duct acoustic modes propagation in a subsonic mean flow. There are no entropy wave and vorticity wave incident upon the inflow boundary region except acoustic wave. For simplification, a Newtonian cooling/friction based damping layer buffer zone is introduced at the inflow boundary region. This idea has ever been used as a simple nonreflective BCs. ${ }^{1314}$ The basic governing equation can be written in the following form:

$$
\frac{\partial \theta}{\partial t}+R_{d}^{-1}(x)\left(\theta-\theta_{0}\right)=k(\theta)
$$

where $\theta$ denotes the vector of dependent variables, and the subscript 0 indicates the imposed basic states (i.e., specified incoming wave).

The basic philosophy is to absorb all the incoming disturbances into zero in the buffer zone before they reach to the outer boundary, such as setting $\theta_{0}=0$. This leads to a nonreflective inflow boundary.

\section{Sound Source Boundary}

Assuming an uniform mean flow in the source region, the incoming sound source is prescribed at three grid lines in an additional straight annular duct based on the following formula:

$$
\left(\begin{array}{c}
\varrho^{\prime} \\
u^{\prime} \\
v^{\prime} \\
w^{\prime} \\
p^{\prime}
\end{array}\right)_{m n}=\operatorname{Re}\left\{\left(\begin{array}{c}
N J_{m}\left(\mu_{m n} r\right)+M Y_{m}\left(\mu_{m n} r\right) \\
\frac{k_{x}}{\omega}\left[N J_{m}\left(\mu_{m n} r\right)+M Y_{m}\left(\mu_{m n} r\right)\right] \\
-\frac{i \mu_{m n}}{\omega}\left[N J_{m}^{\prime}\left(\mu_{m n} r\right)+M Y_{m}^{\prime}\left(\mu_{m n} r\right)\right] \\
\frac{m}{r \omega}\left[N J_{m}\left(\mu_{m n} r\right)+M Y_{m}\left(\mu_{m n} r\right)\right] \\
N J_{m}\left(\mu_{m n} r\right)+M Y_{m}\left(\mu_{m n} r\right)
\end{array}\right)\right.
$$

\section{Notation:}

- $J_{m}-m^{t h}$ order Bessel function of the first kind, $Y_{m}$ - Neumann function

- The ratio of $N$ and $M$ is given by the mode number $(m, n)$ and the nondimensional inner radius $r_{i}$ as follows:

$$
\frac{M}{N}=\frac{-J_{m}^{\prime}\left(\mu_{m n} r_{i}\right)}{Y_{m}^{\prime}\left(\mu_{m n} r_{i}\right)}
$$

- The upstream wave number $k_{x}$ in $\mathrm{x}$-direction is then given by the mean flow Mach number $M_{x}$ :

$$
k_{x}=\frac{-\omega M_{x}+\sqrt{\omega^{2}-\left(1-M_{x}^{2}\right) \mu_{m n}^{2}}}{1-M_{x}^{2}}
$$


In general the sound source should also permit the reflected wave from an open end or a turning plane to propagate out without reflection. To serve this role, a transition zone from the source to the interior domain is introduced for forcing the numerical solutions to the analytical sound sources. Here we still follow the idea of Newtonian cooling/friction damping layer as mentioned above. But $\theta_{0}$ is set to the analytical sound source solutions. In this way, the reflected waves from the interior domain are forced to the analytical sound source gradually when they reach the sound source boundary.

\section{Solid Wall Boundary}

At the solid surface a zero normal velocity condition is used to formulate a hard wall $\mathrm{BC}$ for the linear disturbance equations. However, with high order governing equations, the number of boundary conditions required for an unique solution is large. Hence along the curvilinear solid walls, a slip boundary condition with ghost points inside the wall is imposed. ${ }^{15}$

\section{Axisymmetric Boundary}

For the $2.5 \mathrm{D}$ approach a special axial boundary condition is needed. Actually it is not axisymmetric anymore, but the quantities at the lines below the symmetry line are easily obtainable from the assumed dependency on $m$, only the mode number $m$ is needed. Inserting $\phi=\pi$ below the symmetry line one obtains the coefficient $\operatorname{Re}\left\{e^{-i m \pi}\right\}=$ $(-1)^{m}$ for the mirrored points below the axis.

For the velocity components this boundary condition has to be formulated in the coordinate system that is used above the axis. Hence it is not enough to insert $\phi=\pi$, also the sign has to be changed for the $v^{\prime}$ and $w^{\prime}$ velocity component below the axis. The axisymmetric boundary conditions can be expressed as:

$$
\begin{array}{r}
p^{\prime}(-r)=(-1)^{m} p^{\prime}(r) \\
\varrho^{\prime}(-r)=(-1)^{m} \varrho^{\prime}(r) \\
u^{\prime}(-r)=(-1)^{m} u^{\prime}(r) \\
v^{\prime}(-r)=-(-1)^{m} v^{\prime}(r) \\
w^{\prime}(-r)=-(-1)^{m} w^{\prime}(r)
\end{array}
$$

Another problem is the singularity of the cylindrical coordinates at the axis (for $r \rightarrow 0$ ). The partial differential equations contain parts of $p^{\prime}, \varrho^{\prime}$ and $\left(v^{\prime}-i m w^{\prime}\right)$, which are divided by $r$.

From physics is clear, that none of the ratios can approach to infinity, hence this singularity must be discussed for each mode by it's special physics. The axisymmetric mean flow has no component orthogonal to the axis at
Table 2 Singularity treatment $(r \rightarrow 0)$ for different azimuthal modes

\begin{tabular}{lcc}
$m$ & $\frac{i m p_{m}^{\prime}}{r}$ & $\frac{v_{m}^{\prime}-i m w_{m}^{\prime}}{r}$ \\
\hline 0 & $\frac{\partial i 0 p_{m}^{\prime}}{\partial r}=0$ & $\frac{\partial v_{m}^{\prime}}{\partial r} \neq 0, v_{m}^{\prime}=0$ \\
\hline 1 & $\frac{\partial i p_{m}^{\prime}}{\partial r} \neq 0$ & $\frac{\partial v_{m}^{\prime}-i w_{m}^{\prime}}{\partial r}=0$ \\
& $i p_{m}^{\prime}=0$ & $v_{m}^{\prime}-i w_{m}^{\prime}=0$ \\
\hline 2 & $\frac{\partial i 2 p_{m}^{\prime}}{\partial r}=0$ & $\frac{\partial v_{m}^{\prime}-2 i w_{m}^{\prime}}{\partial r} \neq 0$ \\
& $i 2 p_{m}^{\prime}=0$ & $v_{m}^{\prime}=w_{m}^{\prime}=0$ \\
\hline$m>2$ & $\frac{\partial i m p_{m}^{\prime}}{\partial r}=0$ & $\frac{\partial v_{m}^{\prime}-i m w_{m}^{\prime}}{\partial r}=0$ \\
\hline
\end{tabular}

the singularity. The axially symmetrical subsonic mean flow even forces $\bar{V}$ to vanish faster then $r$, therefore the terms $\frac{1}{r} \bar{V} \varrho_{m}^{\prime}$ and $\frac{1}{r} \bar{V} w_{m}^{\prime}$ are zero at the axis. Only two terms remain to be considered. A simple way is to approximate $1 / r$ by $\partial / \partial r$. This must be discussed for different azimuthal modes.

For the $m=1$ mode the value of the velocity components is non-zero at the singularity. The real part of $v^{\prime}$ and the imaginary part of $w^{\prime}$ describe the same velocity component, but the direction is reverse. To avoid numerical trouble, this derivative should be neglected for $m=1$. The only part that must be replaced by its derivative is the pressure divided by $r$. If the value of the quantity to be divided by $r$ is zero at the axis, it can be repaced by the derivative in terms of $r$.

The $m=0$ and $m=2$ modes are treated in the same manner, omitting any part of the equation which is not necessarily to be divided by $r$, because it has to be zero, or it's limitation has to be zero. Higher modes are always behaving like the $m^{\text {th }}$ order Bessel function of the first kind at the axis. This special treatment of each mode $m$ are shown in table 2.

\section{$\underline{\text { RESULTS AND DISCUSSION }}$}

\section{VALIDATION BY HARD WALLED CIRCULAR}

\section{AND ANNULAR DUCTS}

The CAA procedure is firstly validated by a straight circular and a straight annular hard walled infinite duct with acoustic modes propagation against subsonic uniform mean flows. A sketch of the arrangement of boundary conditions for a circular geometry is described in Fig.1. For the annular duct case, the axisymmetric boundary condition is replaced by the solid wall boundary condition. In both cases, the sound sources are excited against uniform mean flow with $M a=-0.5$. Since the cross section and mean flow are constant for both validation cases, there are no reflection waves from the inflow regions. Hence the numerical results can be compared with analytical upstream 


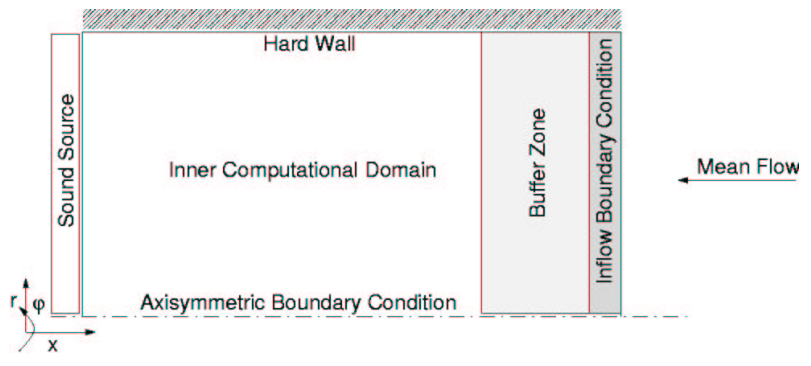

Figure 1 Boundary conditions for a circular duct.

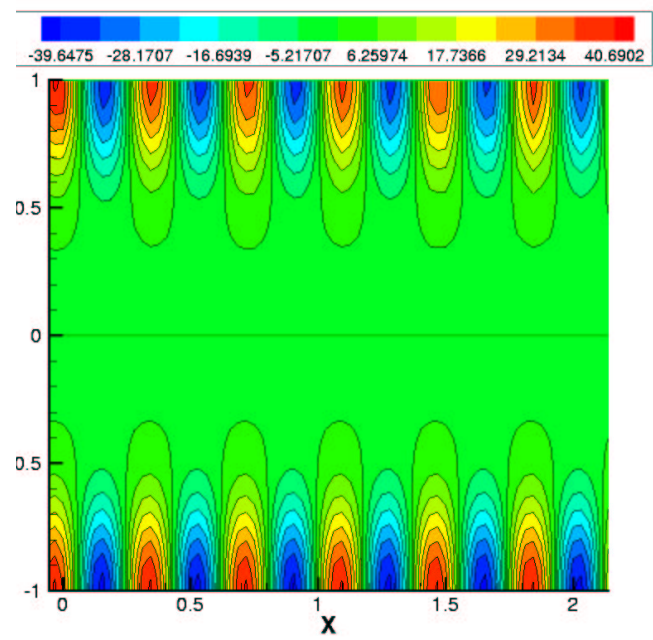

Figure 2 Instantaneous pressure contours in a circular duct, $m=6, n=1, \omega=10, M a=-0.5$.

wave solutions.

In the circular duct case, a uniform grid with $\Delta x=$ $\Delta r=1 / 32$ is distributed in the interior domain along both the $x-$ and $r-$ directions. The grids are stretched in the $x$-direction in the buffer zone. The total number of grid points used in the computation is $120 \times 37$. Fig. 2 give the comparison between numerical and analytical solutions for $m=6, n=1, \omega=10$. There is a good agreement of the numerical and the analytical results.

For the annular duct case, the outer wall radius is $r_{2}=1$ whereas the inner wall radius is $r_{1}=0.42356$. Uniform grids are distributed along both the $x$ and $r$ directions in the interior domain. The grid is stretched in the $x$-direction to obtain a longer buffer zone at low computational costs. The total grid number is 120 . The comparison between CAA and analytical solutions at $m=10, n=1, \omega=16$ is shown in Fig.3. Again the numerical results agree well with the analytical solutions.

\section{ACOUSTIC DUCT MODE PROPAGATION IN AN}

\section{AXISYMMETRIC AERO-ENGINE DUCT INLET}

For furtherly showing the capability of the 2.5D CAA procedure, an axisymmetric inlet with aero-engine like geometry from the paper of Rienstra \& Eversman ${ }^{8}$ is selected

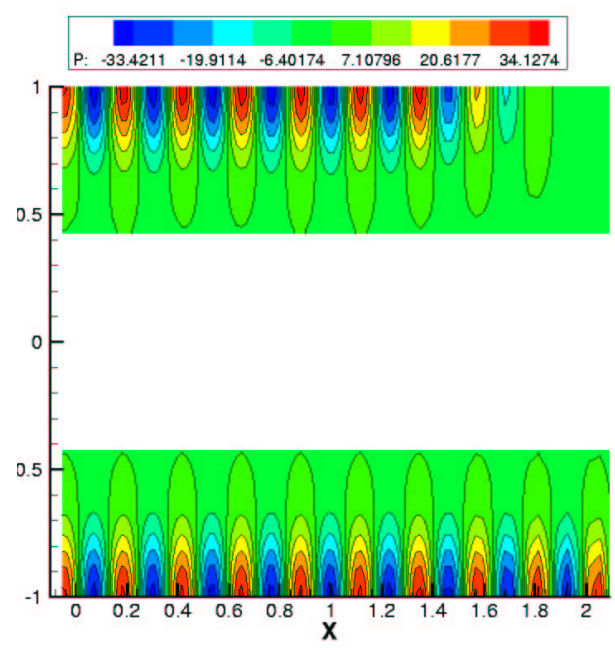

Figure 3 Instantaneous pressure contours in an annular duct, $m=10, n=1, \omega=10, M a=-0.5$.

for benchmarking. The outer and inner wall radius $r_{2}$ and $r_{1}$ are described by

$$
\begin{aligned}
& r_{2}(x)=1-0.18453 x^{\prime 2}+0.10158 \frac{e^{-11\left(1-x^{\prime}\right)}-e^{-11}}{1-e^{-11}} \\
& r_{1}(x)=\max \left[0,0.64212-\left(0.04777+0.98234 x^{\prime 2}\right)^{\frac{1}{2}}\right]
\end{aligned}
$$

where $x^{\prime}=x / L, L=1.86393, r_{1}(0)=0.42356$, spinner tip at $x=1.13547$.

A sketch inlet geometry together with the prescribed boundary conditions for the CAA simulation is depicted in Fig.4. Body fitted grids are generated with $451 \times 151$ points in $x$ and $r$ direction, respectively. In addition a buffer zone is introduced from $x=1.86393$ to 3.0 to absorb incoming disturbances. For reducing computation time, the domain was split into evenly distributed multiblocks for parallel computations.

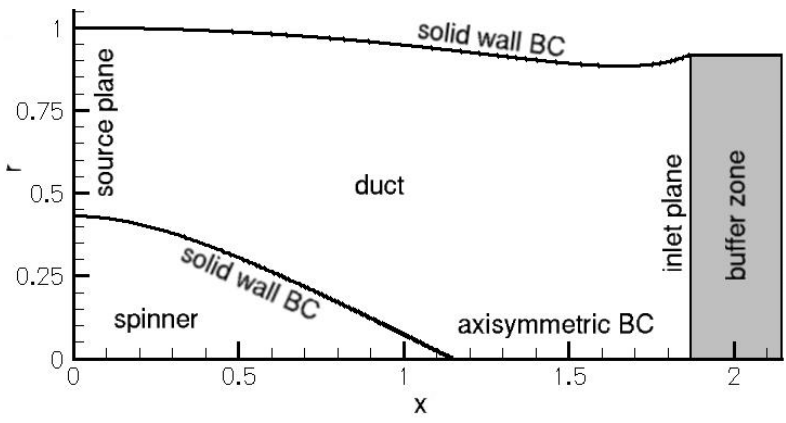

Figure 4 Boundary conditions for a generic aero-engine inlet geometry

\section{Mean Flow Field}

The mean flow field, the geometry, as well as the boundary conditions are assumed to be axisymmetric for this test 

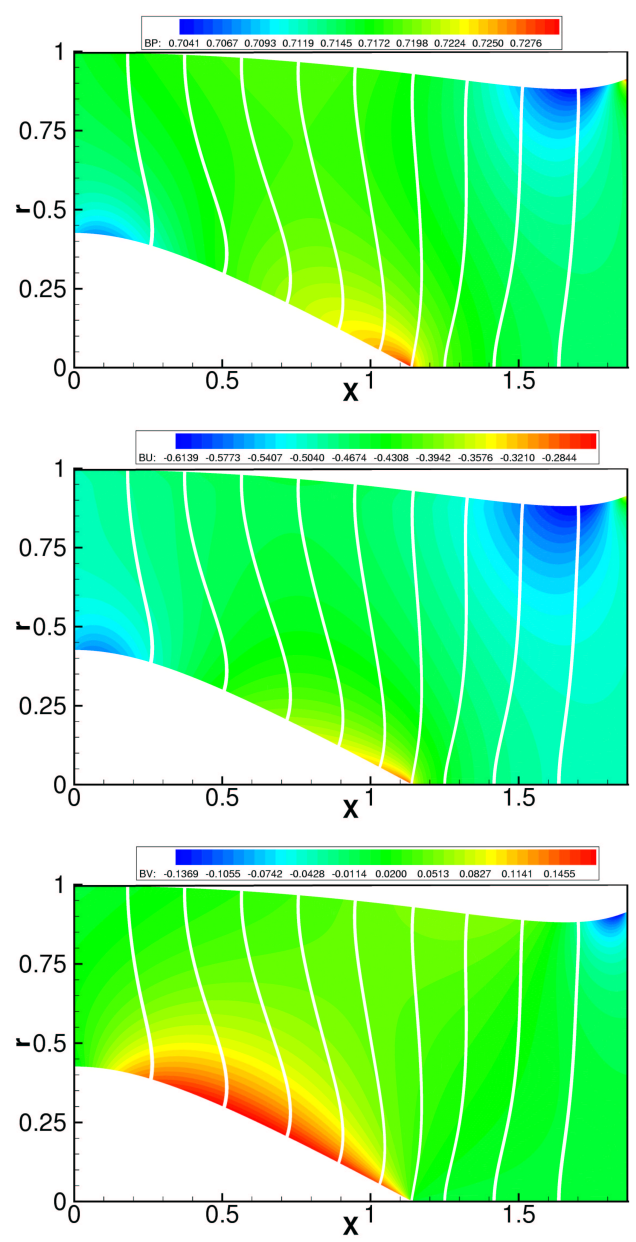

Figure 5 Normalized mean flow contours, CFD Euler solution, $M a(x=0)=-0.5$. Top down: normalized pressure, and the velocity components $\bar{U}$ and $\bar{V}$ (White lines mark block borders of the acoustic grid for parallel processing)

case. The mean flow is assumed two-dimensional, so that there is no swirling component in the mean flow $(\bar{W}=0)$. A low order CFD Euler solution was used as the basing mean flow for the computation of sound propagation as shown in Fig.5.

Because of different requirements for CFD and CAA calculations both procedures need different grids. This requires an interpolation of the mean flow field from the CFD grid to the CAA grid. This routine was provided by DLR. ${ }^{16}$ It can be seen clearly that the mean flow varies in both radial and axial directions. This is different from the quasi one-dimensional potential mean flow used in the FEM and MS computations. ${ }^{8}$ Since the analytical sound source requires a uniform mean flow, this difference results in the inconsistence at the source boundary region in the CAA computations. For this reason, unsmoothed numerical solutions should be expected to be observed in the current numerical results. This situation can be alleviated through the introduction of a long staight annular duct transition zone from the sound source plane to the section of variable area. But all the results presented below were just calculated based on a non-uniform mean flow, in which the sound source is located at $x=0$ plane.

\section{Acoustic Field}

Firstly, the numerical results for $m=10, n=1, \omega=16$ without mean flow case is given in Fig. 6 and compared with the solutions of FEM and MS methods. ${ }^{8}$ The main pattern of the CAA results agree rather well with the FEM and MS solutions. However, the CAA solutions are much more close to the FEM solutions. The main reason is that both the CAA and FEM numerical methods permit the propagation of many modes and scattering is part of the numerical solutions. While the MS method assumes that a single mode does not scatter into other modes in the slowly varying duct. The difference between the CAA and FEM solutions near the boundary region can be attributed to the different treatment of boundary conditions.
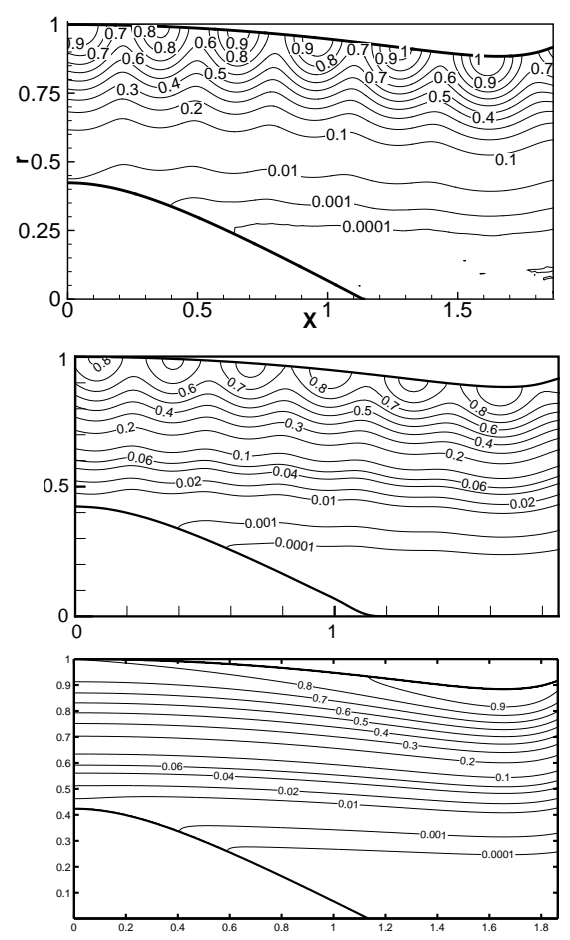

Figure 6 Normalized pressure contours $(M a=0, m=$ 10, $n=1, \omega=16)$, Top down: CAA, FEM, MS

For the same case, but with mean flow at $M a=-0.5$, Fig.7 gives the results ( $m=10, n=1, \omega=16$ ). The results show a good agreement between CAA and FEM, as well as between CAA and MS. Some visible wiggles in the CAA and FEM solutions are probably due to the inherent reflection from the lip region and inlet plane. The 

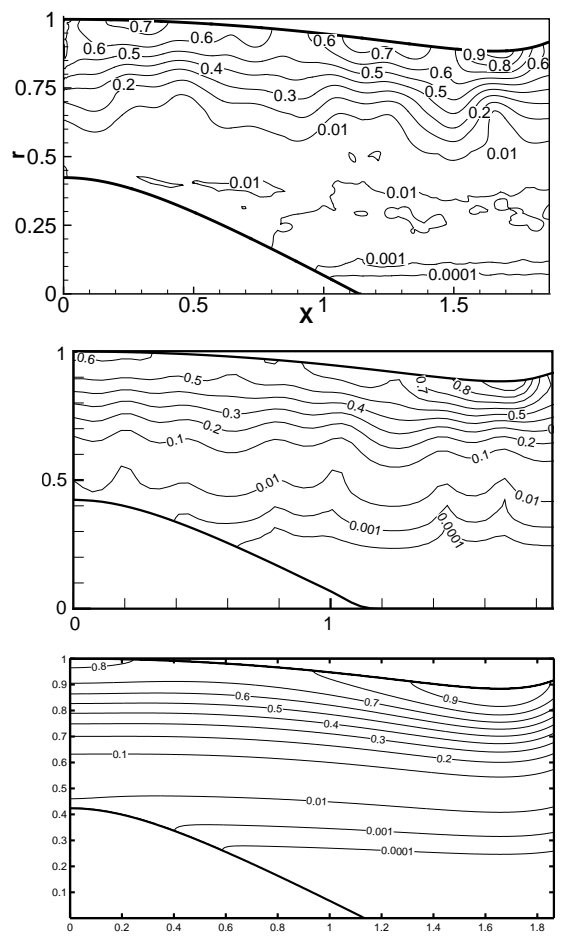

Figure 7 Normalized pressure contours $(M a=-0.5, m=$ 10, $n=1, \omega=16$ ), Top down: CAA, FEM, MS

MS and FEM show more smooth results in the spinner and near source inner wall regions. This difference can be the result of high gradient mean flows in these regions, see Fig. 5 while FEM and MS solutions were based on quasione dimensional mean flows.

For high frequency cases $(M a=-0.5, m=10, n=1$ and $\omega=50$ ), many radial modes are cut on, see the comparison results in Fig. 8. There is interference with higher radial modes since $n \leq 11$ is cut on for this case. Although the general agreement is good, wiggles can be observed in the source region of CAA solutions. This can be partly due to the inconsistence of the uniform mean flow assumption of the sound source with the radially varying Euler mean flow used in CAA computations.

For higher azimuthal mode cases of $m=20$ and $m=30$, the comparisons are shown in Fig. 9 and Fig. 10, respectively. The numerical solutions(CAA, FEM) agree better with the MS solutions comparing with lower azimuthal mode case of $m=10$. However, the wiggles in the CAA and FEM solutions are still visible because of the shift to higher $n$ modes. Unsmoothed wiggles can also be found in the source region of CAA solutions due to radially and axially nonuniform Euler mean flow in the source region.

For higher radial mode cases, two numerical results of instantaneous pressure contours are shown in Fig. 11 for
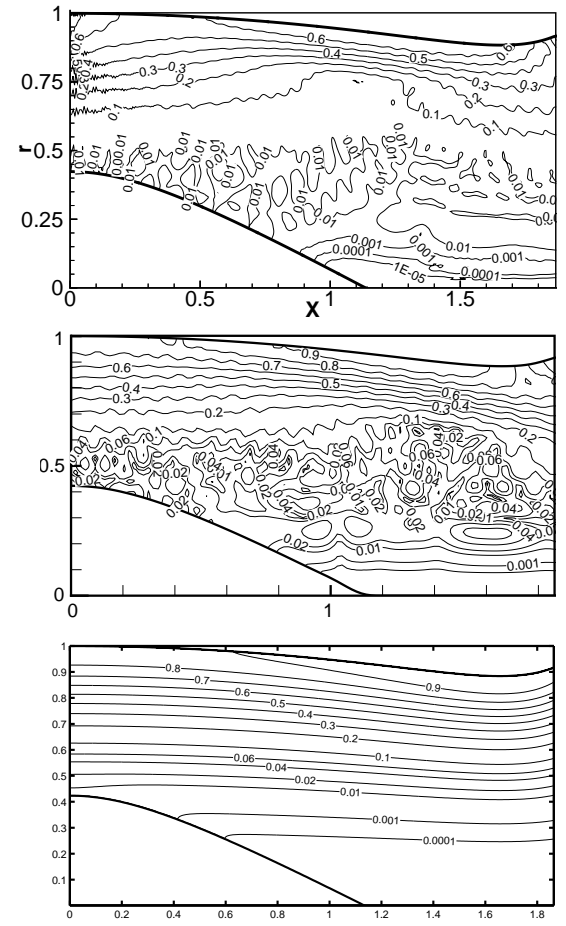

Figure 8 Normalized pressure contours $(M a=-0.5, m=$ $10, n=1, \omega=50)$, Top down: CAA, FEM, MS
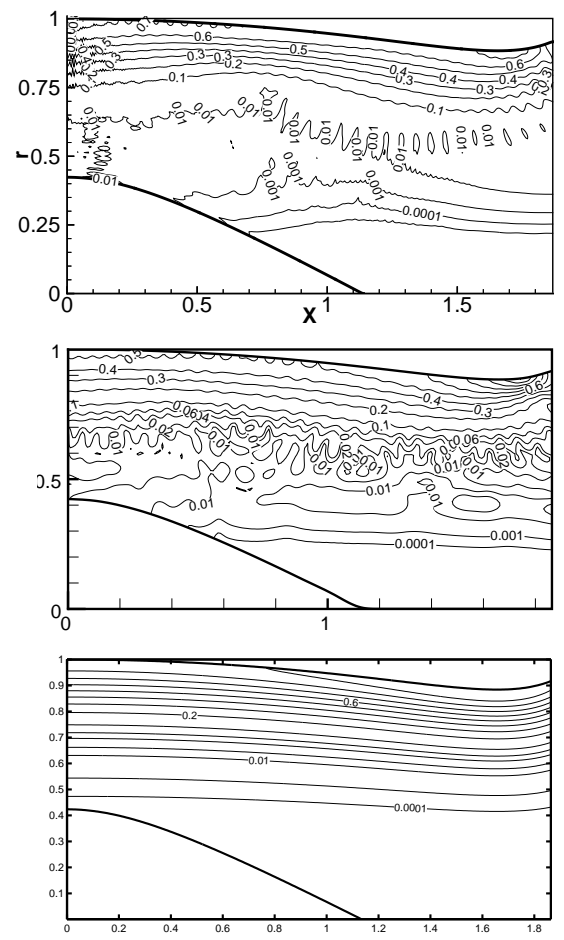

Figure 9 Normalized pressure contours $(M a=-0.5, m=$ 20, $n=1, \omega=50)$, Top down: CAA, FEM, MS

( $m=10, n=12, \omega=50)$ and $(m=20, n=5, \omega=50)$. The inteference structure and reflection from the solid wall can be clearly observed.

To furtherly show the capability, a cut-on and cut-off 

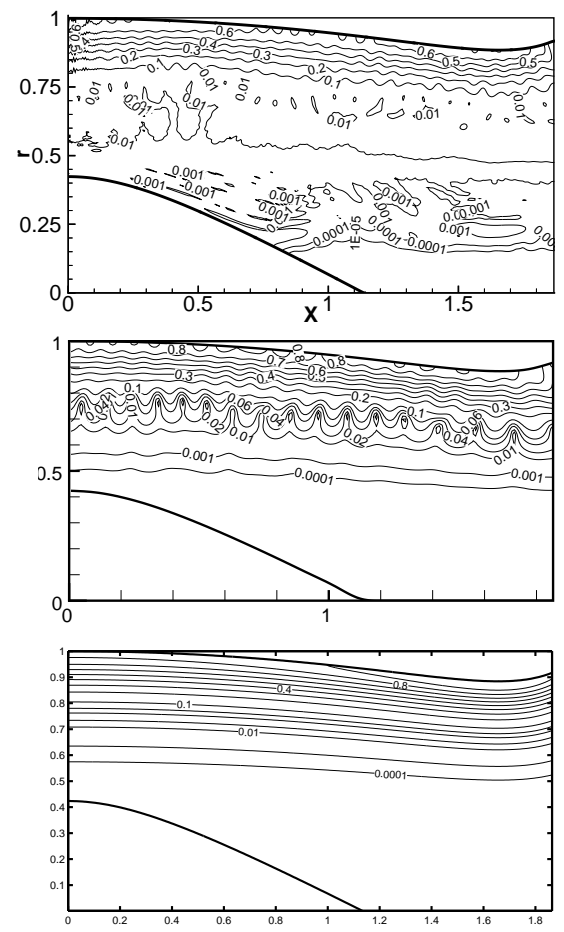

Figure 10 Normalized pressure contours $(M a=-0.5, m=$ 30, $n=1, \omega=50)$, Top down: CAA, FEM, MS
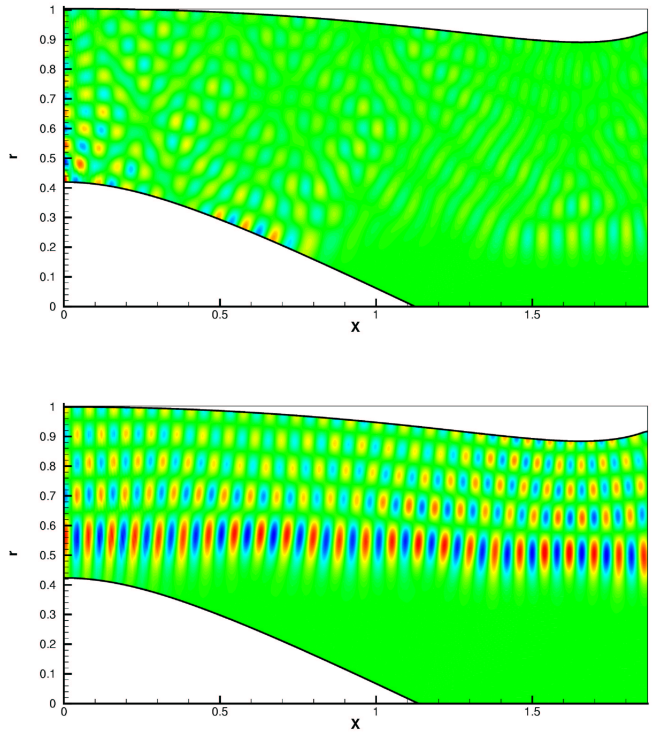

Figure 11 Instantaneous pressure contours for $M a=-0.5$, Top: $(m=10, n=12, \omega=50)$, Lower: $(m=20, n=5, \omega=$ 50)

turning plane case is calculated by the CAA approach. Fig. 12 gives the normalized pressure contours for a spinning mode $M a=-0.5, m=+10, n=1, \omega=11.129$. The reconstructed $3 \mathrm{D}$ pressure contours on the casing surface is shown in Fig. 13. It can be found that the calculated turning plane is located around $x \approx 0.63$ plane. But the turning plane is difficult to detect near the spinner wall region. The main reason is that the cut-on and cut-off phenomenon is very sensitive to the related mean flow field. Differently form MS and FEM, CAA is based on a Euler based mean flow field, hence the CAA calculated transition plane can not be directly compared with that computed by MS and FEM if they will be available in the future. Fig. 14 further gives the sound pressure level (SPL, dB) distribution at four axial planes at $x=0.5,1.0,1.5,1.8$, for an assumed peak sound source amplitude $p_{\max }=1000 \mathrm{~Pa}$.

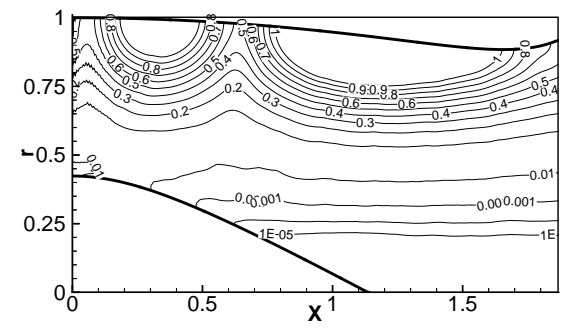

Figure 12 Normalized pressure contours for a cut-on and cut-off transition case $(M a=-0.5, m=10, n=1, \omega=$ 11.129). The turning plane is situated around $x=0.63$.

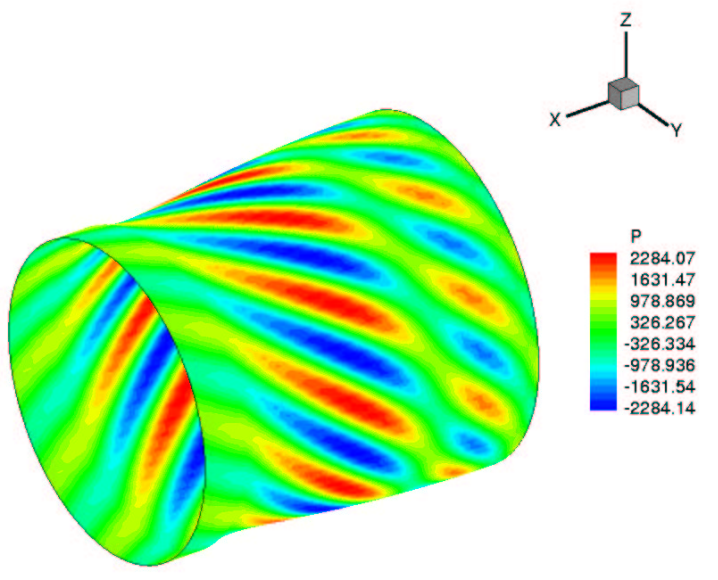

Figure 13 3D reconstructed pressure contours for the cut-on and cut-off transition case $(M a=-0.5, m=+10, n=1, \omega=$ 11.129). 

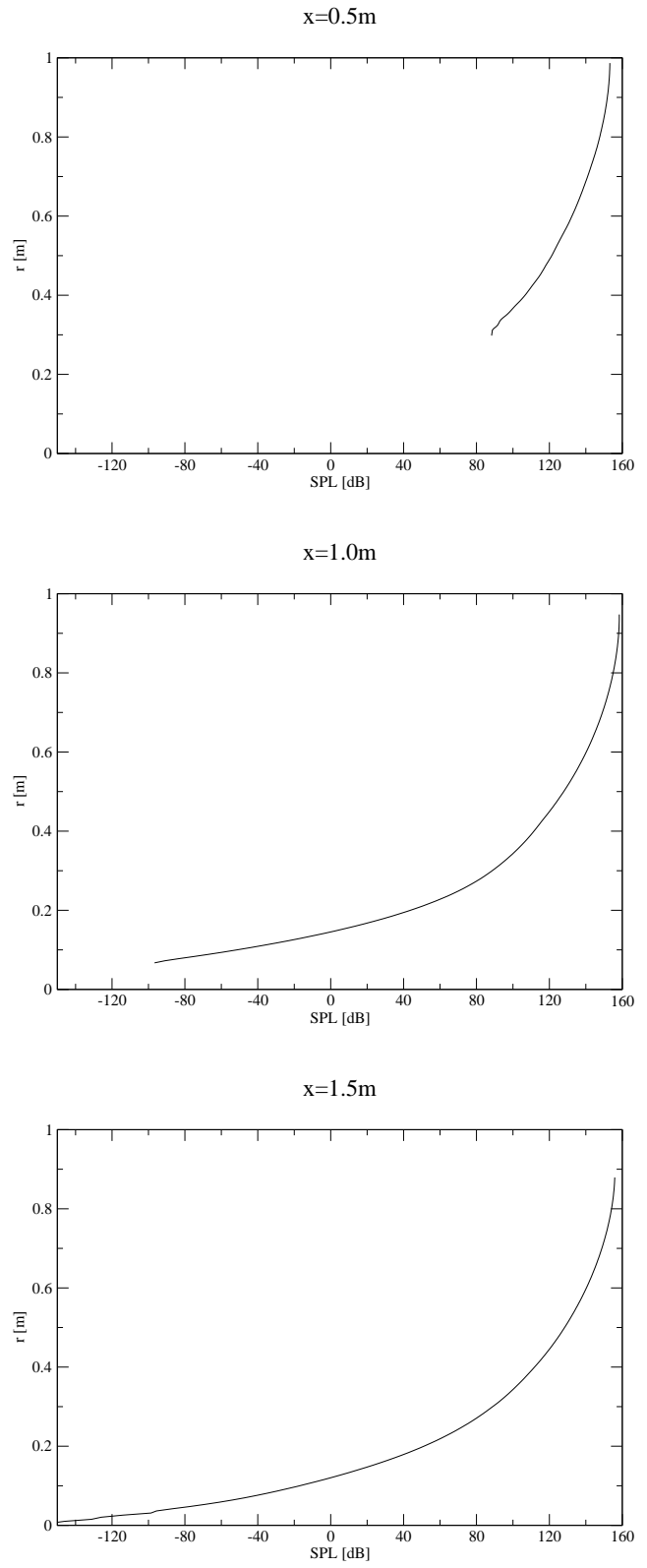

$\mathrm{x}=1.8 \mathrm{~m}$

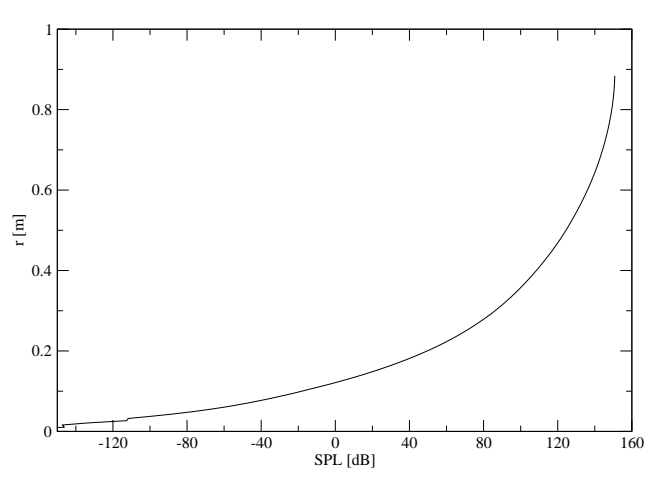

Figure 14 SPL distribution at 4 axial planes for the cut-on and cut-off transition case $(M a=-0.5, m=10, n=1, \omega=$ 11.129).

\section{CONCLUSION}

A 2.5D CAA approach is proposed to simulate sound wave propagation in axisymmetric duct flows. The main idea is to calculate each azimuthal mode using Fouries decomposition technique in circumferiential direction. The optimized high-order DRP finite-difference scheme was employed for spatial discretization and the a low-dissipation and low-dispersion Runge-Kutta scheme was applied for time integration. Numerical validation examples are given for a straight circular duct and a straight annular duct containing uniform subsonic mean flows. For checking the capability of the 2.5D CAA approch, the acoustic propagation in an aero-engine geometry duct inlet is simulated and compared with the FEM and MS solutions. It is shown that the CAA solutions agree reasonably well with the solutions by FEM and MS methods. The main advantages of the proposed 2.5D CAA approach are twofold. One is that this approach has potential to account for more mean flow effects on sound wave propagation compared with the potential mean flow assumed in the current FEM and MS methods. Another advantage is that it is very efficient compared with fully 3D computations for an axisymmetric duct flows. However, fully 3D methods are still desirable for those fully 3D cases, such as scarfed inlets.

\section{ACKNOWLEDGMENT}

This work was partly carried out in the framework of the EC project TurboNoiseCFD and the DFG SWING+ project. The authors appreciate Prof. Rienstra and Prof. Eversman for their kind permission of using their MS and FEM results in this paper for comparison. The first author (X.D. Li) would also like to thank partial support from NSFC-50076002 and ASFC-01C51036.

\section{References}

${ }^{1}$ Ozyoruk, Y., and Long, L.N., "Computation of Sound Radiating from an Engine Inlets," AIAA Journal, Vol.34, No.5, 1996, pp.894-901.

${ }^{2}$ Ait-Ali-Yahia, D., Stanescu, D., Robichaud, M.P. and Habashi, W.G., "Spectral Element Grid Generation and Nonlinear Computations for Noise Radiation from Aircraft Engines," AIAA Paper, 99-1832, 1999.

${ }^{3}$ Ahuja, V., Ozyoruk, Y. and Long, L., "Computational Simulation of Fore and Aft Radiation from Ducted Fans,” AIAA Paper, 2000-1943, 2000

${ }^{4}$ Druault, Ph., Gerolymos, G.A., Nousis, I., "Comparison of Theoretical and Computational Results of Spinning Acoustic Modes Propagation in Aircraft Engine Intakes," 5th CEAS-ASC Workshop on "Turbomachinery Noise and Duct Acoustic", Eindhoven University of Technology, Eindhoven, the Netherlands, 8-9 November 2001.

${ }^{5}$ Eversman, W., Parret, A. V., Preisser, J. S., and Silcox, R. J., "Contributions to the Finite Element Solution of the Fan Noise Radiation Problem," ASME Journal of Vibration, Acoustic, Stress and Reliability in Design, Vol. 107, April 1985, pp. 215-223. 
${ }^{6}$ Rienstra, S.W., "Sound Transmission in Slowly Varying Circular and Annular Ducts with Flow," Journal of Fluid Mechanics, 380, 1999, 279 296.

${ }^{7} \mathrm{Li}$, X.D., Schoenwald, N., Thiele, F. "Numerical Computation of Sound Propagation and Radiation in a Duct," AIAA Paper 2001-2179, 2001.

${ }^{8}$ Rienstra, S.W. and Eversman, W., "A numerical comparison between the multiple-scale and finite-element solution for sound propagation in lined flow ducts," Journal of Fluid Mechanics, 437, 2001, pp.367-384.

${ }^{9}$ Tam, C.K.W. and Webb, C., "Dispersion-Relation-Preserving Finite Difference Schemes for Computational Aeroacoustics," Journal of Computational Physics, 107, 1993, pp.262-281.

${ }^{10} \mathrm{Hu}$, F. Q., Hussani, M. Y., and Manthey, J. L., "Low-dissipation and Low-dispersion Runge-Kutta Schemes for Computational Aeroacoustics," Journal of Computational Physics, 124, 1996, pp.177-191.

${ }^{11}$ Stanescu, D. and Habashi, W.G., "2N-storage low-dissipation and Low-dispersion Runge-Kutta Schemes for Computational Aeroacoustics," Journal of Computational Physics, 143, 1998, pp.674-681.

${ }^{12}$ Tam, C.K.W., Webb, C. and Dong, T.Z., "A Study of Short Wave Components in Computational Aeroacoustics," Journal of Computational Aeroacoustics, 1, 1993, pp.1-30.

${ }^{13}$ Li, F., Tam, C.K.W., Choudhari, M., "Numerical boundary conditions for simulation of gust-cascade interaction," AIAA-CEAS Paper 99-1845, 1999.

${ }^{14}$ Israeli, M. and Orszag, S.A., "Approximation of radiation boundary condition," Journal of Computational Physics, 41, 1981, pp.115-135.

${ }^{15}$ Tam, C.K.W. and Dong, Z., "Wall Boundary Conditions for High-Order Finite Difference Schemes in Computional Aeroacoustics," Theroretical and Computional Fluid Dynamics, 8(6), 1994, pp.303-322.

${ }^{16}$ Lummer, M., Groggers, H.A., Delfs, J.W. "Using Rans Mean Flow Fields in Numerical Aeroacoustics Simulation (CAA)" NATO RTO AVT Symposium on Ageing Mechanism and Control, Part A- Developments in Computational Aero - and Hydro - Acoustics, Manchester, UK, 8-11 October 2001 